Review

\title{
Host microenvironment in breast cancer development Inflammatory cells, cytokines and chemokines in breast cancer progression: reciprocal tumor-microenvironment interactions
}

\author{
A Ben-Baruch \\ Department of Cell Research and Immunology, George S Wise Faculty of Life Sciences, Tel-Aviv University, Israel \\ Corresponding author: A Ben-Baruch (e-mail: aabb@post.tau.ac.il)
}

Received: 19 August 2002 Revisions received: 8 October 2002 Accepted: 8 October 2002 Published: 28 October 2002

Breast Cancer Res 2003, 5:31-36 (DOI 10.1186/bcr554)

(C) 2003 BioMed Central Ltd (Print ISSN 1465-5411; Online ISSN 1465-542X)

\begin{abstract}
A comprehensive overview of breast cancer development and progression suggests that the process is influenced by intrinsic properties of the tumor cells, as well as by microenvironmental factors. Indeed, in breast carcinoma, an intensive interplay exists between the tumor cells on one hand, and inflammatory cells/cytokines/chemokines on the other. The purpose of the present review is to outline the reciprocal interactions that exist between these different elements, and to shed light on their potential involvement in breast cancer development and progression.
\end{abstract}

Keywords: inflammatory cells, inflammatory cytokines/chemokines, tumor necrosis factor alpha

\section{Introduction}

It has long been recognized that the interactions of tumor cells with their microenvironment may affect tumor growth and metastasis formation. The tumor microenvironment may differ between tumor types and disease stages, is complex, and consists of many cell types and factors. Of these, inflammatory cells and cytokines were recently suggested to play a key role in breast carcinoma. A large number of observations suggest that certain types of inflammatory cells are not innocent bystanders at breast tumor sites, and that they actively affect tumor development and progression. Inflammatory cells, primarily macrophages, may affect these processes via their ability to express a large variety of factors, including inflammatory cytokines. These cytokines may be secreted not only by inflammatory cells, but also by the tumor cells and stroma cells, together establishing a network of factors that significantly affects breast cancer.

A short review is presented on the activities of inflammatory cells and inflammatory cytokines in breast carcinoma, illuminating the multifaceted abilities of these factors to affect the progression of this disease. In addition, the present review will provide an outline of the role of inflammatory chemokines in breast carcinoma. The ability of inflammatory cells, cytokines and chemokines to affect tumor cell-microenvironment interactions will be illustrated, providing evidence for the activities of these factors in breast cancer development and progression.

\section{Inflammatory cells and cytokines in breast cancer}

Breast carcinomas are heavily infiltrated by different types of host leukocytes, including primarily $\mathrm{T}$ cells, and monocytes that differentiate into tumor-associated macrophages (TAM) at the tumor site [1-7]. The presence of the cellular infiltrate in breast tumors was initially regarded as evidence for the potential activity of immune mechanisms against the growing neoplasm. There are an increasing number of studies, however, suggesting that T-cell antitumor responses are impaired in advanced stages of breast carcinoma. On the whole, there is no definite conclusion regarding the efficacy of T-cell-dependent immune mechanisms, or regarding the correlation between the extent/type of T-cell infiltration and tumor progression in most subtypes of carcinoma of the breast $[1,2,7]$. The only exception is the relatively infrequent type of medullary carcinoma, in which favorable prognosis was correlated with intensive lymphoid infiltration $[1,2]$. 
Table 1

\begin{tabular}{|c|c|}
\hline Promalignant activities & References \\
\hline \multicolumn{2}{|l|}{ TAM-derived factors* } \\
\hline Growth factors & {$[5-8]$} \\
\hline Proteases & {$[3,5,7,8]$} \\
\hline Angiogenic mediators & {$[3-5,7,8,20]$} \\
\hline $\begin{array}{l}\text { Promalignant chemokines (CCL2, CXCL8; } \\
\text { CCL5 - by unidentified leukocytes) }\end{array}$ & {$[5,18,22]$} \\
\hline Reactive oxygen intermediates & {$[3,5]$} \\
\hline TNF- $\alpha$ & {$[5,7,8,10,11]$} \\
\hline $\begin{array}{l}\text { Immunosuppressive mediators }\left(\mathrm{PGE}_{2}, \mathrm{IL}-10 \text {, }\right. \\
\text { TGF- } \beta \text { ) }\end{array}$ & {$[3,5,8]$} \\
\hline Others & [5] \\
\hline \multicolumn{2}{|l|}{ TAM-derived TNF- $\alpha$ may promote the expression of: ${ }^{*}$} \\
\hline $\begin{array}{l}\text { Promalignant chemokines (e.g. CCL5 by } \\
\text { breast tumor cells) }\end{array}$ & {$[8,9,25,27]$} \\
\hline $\begin{array}{l}\text { Matrix metalloproteinases, proteases } \\
\text { (e.g. by breast tumor cells) }\end{array}$ & {$[8,9,25]$} \\
\hline Reactive oxygen intermediates & {$[8,9]$} \\
\hline Angiogenic mediators & {$[1,8,9]$} \\
\hline Others & {$[1,8,9]$} \\
\hline \multicolumn{2}{|l|}{$\begin{array}{l}\text { Tumor cell-derived and leukocyte-derived CCL5 } \\
\text { and CCL2 may promote: }\end{array}$} \\
\hline Monocyte migration to breast tumors & {$[18-20,25]$} \\
\hline $\begin{array}{l}\text { Matrix metalloproteinase expression by } \\
\text { monocytic and breast tumor cells }\end{array}$ & {$[25,26]$} \\
\hline $\begin{array}{l}\text { Angiogenesis/vascularization (may also be } \\
\text { induced by CXCL8) }\end{array}$ & $\begin{array}{l}{[18,19,21} \\
25,32,34]\end{array}$ \\
\hline
\end{tabular}

TAM, tumor-associated macrophages; TNF- $\alpha$, tumor necrosis factor alpha; $P \mathrm{PE}_{2}$, prostaglandin $\mathrm{E}_{2}$; IL, interleukin; TGF- $\beta$, transforming growth factor beta. ${ }^{*}$ In general, some in breast carcinoma.

In contrast to T lymphocytes, a large body of evidence suggests that high levels of TAM are correlated with poor prognosis in breast carcinoma. Many studies have shown a positive relationship between high levels of TAM and lymph node metastases in breast carcinoma, and suggested that the density of TAM is associated with clinical aggressiveness [1,4-7]. The potential contribution of TAM to breast carcinoma progression was initially unexpected, in view of several potential antimalignant activities that may be exerted by these cells, including antigen presentation, cytotoxicity and phagocytosis [3]. Solid evidence has, however, suggested the promalignant activities of TAM in breast carcinoma [4-7]. The tumor-promoting activities of TAM may be the result of their ability to express numerous tumor-promoting characteristics, such as growth factors for breast tumor cells, angiogenic mediators, extracellular matrixdegrading enzymes and inflammatory cytokines (Table 1) [3-8]. It was also suggested that TAM might contribute to tumor progression by the release of reactive oxygen intermediates $[3,5]$. These products may induce mutagenic changes that could result in increased DNA damage and generation of diversity within the tumor $[3,8,9]$.
A major TAM-derived inflammatory cytokine shown to be highly expressed in breast carcinomas is tumor necrosis factor alpha (TNF- $\alpha)[5,8,10,11]$, which is a multifactorial cytokine. As implied by its name, TNF- $\alpha$ may have cytotoxic and apoptotic activities when administered to breast tumor cell lines. However, these effects may depend on multiple factors, such as treatment by estrogen and the expression of members of the epidermal growth factor receptor family.

The fact that TNF- $\alpha$ activities vary under different physiological conditions and in a cell-type-dependent manner contributes to a sense of ambiguity regarding its antitumor effects $[8,9]$. Indeed, recent investigations strongly suggest that the chronic expression of TNF- $\alpha$ in breast tumors actually supports tumor growth. The number of cells expressing TNF- $\alpha$ in inflammatory breast carcinoma was found to be correlated with increasing tumor grade and node involvement, and TAM-derived TNF- $\alpha$ expression was suggested to play a role in the metastatic behavior of breast carcinomas $[10,11]$. Furthermore, patients with more progressed tumor phenotypes were shown to have significantly higher TNF- $\alpha$ serum concentration [12].

The tumor-promoting functions of TNF- $\alpha$ may be mediated by its ability to induce proangiogenic functions, to promote the expression of matrix metalloproteinases (MMP) and endothelial adhesion molecules, and to cause DNA damage via reactive oxygen, the overall effect of which is promotion of tumor-related processes (Table 1) $[1,8,9]$.

The role of two other inflammatory cytokines (possibly TAM derived [3,5]), IL-6 and IL-1, was also addressed in breast carcinoma. Several contradicting in vitro studies were recently followed by an analysis of the expression of IL-6 and IL-1 in biopsies, tumor homogenates and/or serum. Although these recent studies suggest that elevated levels of IL- 6 may contribute to disease progression, a definite conclusion in this issue has not yet been reached (for example $[13,14])$. Initial analyses regarding IL-1 $\beta$ indicated that its levels were significantly higher in invasive carcinoma than in ductal carcinoma in situ or in benign lesions, implying that elevated levels of IL-1 $\beta$ are directly correlated with a more advanced disease [15]. Of interest is the fact that the two cytokines (IL-6 and IL-1) and TNF- $\alpha$ are interrelated and may act in an additive manner, suggesting that these three cytokines form a network of related factors that may affect tumor cell progression in a cooperative manner.

\section{Chemokines in breast cancer}

Chemokines are leukocyte chemoattractants that are divided structurally into four subgroups, based on the composition of cysteine residues in their amino-terminal portion. Another, although less stringent, classification divides the chemokines into two functional subgroups, the 
inflammatory and the homeostatic. The inflammatory chemokines promote leukocyte infiltration to sites of inflammation and their expression is inducible, primarily, by proinflammatory cytokines. On the other hand, the homeostatic chemokines are constitutively expressed and regulate the directed migration of leukocytes in processes of hematopoiesis and lymphoid organ development [16]. Mainly the chemokines that are classically defined as 'inflammatory', but also the 'homeostatic' chemokines, were shown to play a role in various aspects of breast malignancy.

In the limits of the present review, only the role of inflammatory chemokines will be thoroughly discussed. It is important, however, to briefly describe the role of the homeostatic chemokines in breast carcinoma. Breast tumors establish distant, albeit site specific, metastases that have major importance for disease progression. Many stages of the metastatic process resemble those of leukocyte homing to specific organs, raising the possibility that chemokines which are constitutively expressed at breast tumor metastatic sites direct the migration of the tumor cells to these organs. Indeed, it has been shown that target organs for breast metastases are enriched in the chemokine CXCL12 (SDF-1), inducing the specific migration of breast tumor cells that express the relevant receptor for this chemokine, CXCR4 [17]. A similar role in the metastatic spread of breast tumor cells was suggested for the chemokine receptor CCR7 and its corresponding ligands. Of major importance was the observation that neutralizing antibodies against CXCR4 reduced in vivo experimental and spontaneous metastasis formation by breast tumor cells in a transplantable model [17], suggesting that the CXCR4-CXCL12 axis plays a key role in the metastatic process of breast tumors.

\section{Inflammatory chemokines as inducers of leukocyte recruitment to breast tumors}

The potential contribution of TAM to breast cancer progression motivated researchers to analyze the role of monocyte-attracting chemokines, such as CCL2 (MCP-1) and CCL5 (RANTES), in the progression of breast carcinoma. The release of such chemokines by breast tumor cells may mediate the migration of monocytes from the circulation to breast tumors. Studies on these chemokines provided evidence for their potential contribution to breast cancer progression. Several investigations have indicated that CCL2 was highly expressed by the tumor cells as well as by stromal cells (including TAM), and that CCL2 concentration was correlated significantly with TAM accumulation. Furthermore, it was also indicated that high expression of CCL2 was a significant indicator of early relapse and poor prognosis [18-20]. The potential contribution of CCL2 to breast cancer progression was further supported by a study demonstrating that the ability of CCL2-expressing human breast carcinoma MDA-231 cells to form lung micrometastases in mice was significantly inhibited by neutralizing antibodies to CCL2 [21].

In addition to CCL2, high levels of CCL5 were shown to considerably correlate with advanced breast carcinoma [22]. Higher incidence and higher intensity of CCL5 expression were observed in tumor cells in biopsies of patients with advanced breast carcinoma. In contrast, the expression of CCL5 was rarely detected in normal duct epithelial cells and in biopsies of healthy patients (mostly patients diagnosed with benign breast disorders) [22]. CCL5 expression by leukocytes in breast tumors was also noted [22]. Similar observations on the expression of CCL5 in breast carcinoma were made in another study, demonstrating that high CCL5 tissue and plasma levels were correlated with advanced stages of disease [23]. The potential promalignant role of CCL5 in breast carcinoma was supported by findings demonstrating that the overexpression of CCL5 in breast tumor cells resulted in increased invasiveness [24]. It was suggested, therefore, that CCL5 may be involved in breast cancer progression, and may also be used as a prognostic factor in this disease.

The roles of CCL2 and CCL5 in breast cancer progression may be mediated primarily by their ability to induce monocyte migration to tumor sites, as indeed was suggested by findings showing that breast tumor cell-derived CCL2 and CCL5 induced the migration of monocytic cells (Table 1) [25]. Tumor-infiltrating monocytes may be stimulated by chemokines to secret protumorigenic factors, as indicated by the CCL2-induced and CCL5-induced promotion of MMP9 expression by monocytic cells $[25,26]$. In addition, the infiltrating monocytes at the tumor site may now express other factors that may assist in tumor progression, including inflammatory cytokines such as TNF- $\alpha$. These cytokines may further increase the expression of protumorigenic properties by the tumor cells (as shown for MCF-7 and T47D cells), such as MMP2 and MMP9 [25], as well as the release of monocyte-attracting chemokines. Indeed, the expression of CCL5 was shown to be elevated by TNF- $\alpha$, alone or in synergism with IFN- $\gamma$, in different cell lines of breast carcinoma $[25,27]$. TNF- $\alpha$ was also suggested to promote CCL2 expression by tumor cells [8]. In addition, CCL2 release from breast carcinoma cells was promoted by IL- $1 \alpha$ stimulation [28].

The inflammatory chemokines may act not only on the inflammatory cells to increase their protumorigenic properties, but also directly on the tumor cells through specific chemokine receptors that are expressed by these cells (Table 1) $[25,29]$. This was indicated by the ability of CCL5 to promote MMP9 expression by breast tumor cells [25]. Overall, these results suggest that the recruitment of inflammatory cells may be followed by their ability to express promalignant factors (e.g. MMP), as well as 
inflammatory cytokines (TNF- $\alpha$ ) that further increase the expression of chemokines. Elevated levels of chemokines can then further stimulate the attraction of inflammatory cells (such as monocytes), resulting in a 'vicious circle' of continuous infiltration and expression of promalignant factors.

In contrast to CCL2 and CCL5, there are few reports suggesting that chemokine activities may result in recruitment of leukocytes that inhibit breast cancer progression. These studies are based on tumor cells that were transfected to overexpress inflammatory or homeostatic chemokines, primarily the well-described angiostatic chemokines CXCL10 and CXCL9 (IP-10 and Mig, respectively), and also CCL19 (ELC). Depending on the chemokine type, infiltration of $\mathrm{CD}^{+}$cells and/or natural killer cells to tumor sites was observed, and resulted in reduced tumor formation [30,31]. As these systems are based on transplantable tumors, it is as yet difficult to assess the role of these antitumorigenic chemokines in disease progression in breast cancer patients. Nevertheless, these observations pose therapeutic possibilities and indicate that various chemokines may differently regulate breast cancer development, depending to a large extent on the type of leukocytes they attract.

\section{Inflammatory chemokines as inducers of angiogenesis and increased vascularity}

Both CCL2 and CCL5 were suggested to affect the metastatic potential of breast tumor cells by increasing angiogenesis and vascularity. Increased CCL2 expression was correlated with elevated angiogenesis in breast cancer. The ability of CCL2 to induce chemotaxis of human endothelial cells and formation of blood vessels has been described, as well as the ability of CCL5 to promote vascularity in chick chorioallantoic membrane assays $[18,19,21,25]$. In addition to a direct effect of the chemokines on these functions, they may affect angiogenesis indirectly by inducing the recruitment of monocytes to the tumors. CCL2 expression in breast cancer patients was highly associated with the presence of TAM and with the expression of potent angiogenic factors, including vascular endothelial growth factor and others [20]. Because TAM may be an important source of these angiogenic factors, these results imply that CCL2 expression indirectly results in promoted angiogenesis through production of angiogenic factors by TAM.

In addition to CCL2 and CCL5, inflammatory chemokines that belong to the structural subgroup of $E L R^{+}-C X C$ chemokines (CXC chemokines that express an ELR motif) were described as highly potent angiogenic factors [32]. CXCL8 (IL-8), the prototype chemokine of this subgroup, was shown to be highly expressed in breast carcinoma by the tumor cells as well as by stroma cells, and its expression in breast tumor cells required stimulation by the inflammatory cytokines TNF- $\alpha$ or IL-1 $\beta$ [33-35]. Although expressed at high levels in breast tumors, strong evidence for IL-8 correlation with lymph node metastasis and progression in breast cancer was not obtained. However, IL-8 expression by breast tumor cell lines did suggest a role for IL-8 in the metastatic phenotype of these cells [33]. Receptors for IL-8 were detected not only on breast tumor cells, but also on vessel endothelial cells, suggesting that this chemokine has angiogenic roles in breast carcinoma [34].

\section{Conclusion}

Reciprocal interactions that exist between the breast tumor cells and stroma/inflammatory cells are mediated by inflammatory cytokines and chemokines, and may affect tumor development and progression. Many inflammatory factors, including cells, cytokines and chemokines, may divergently regulate the progression process. However, major emphasis has recently been placed on the potential role of TAM in breast cancer progression, mediated by their ability to express promalignant factors. Monocyte migration to breast tumors was highly correlated with the expression of monocyte-attracting chemokines by the tumor cells and stroma cells. As suggested in Figure 1, these chemokines may act in a cooperative manner to attract leukocytes, primarily monocytes, to tumor sites. This may be followed by chemokine-induced stimulation of monocyte-derived promalignant activities at the tumor site. The result of this process may be a further increased expression of protumorigenic properties by the infiltrating monocytes. Monocyte-derived cytokines, such as TNF- $\alpha$, could intensify chemokine expression by the tumor or stroma cells, as well as other promalignant properties. The resulting positive feedback loop may act in conjunction with other promalignant activities of tumor-derived and inflammatory cell-derived factors, as well as with chemokine-induced site-specific metastasis formation (through CXCL12). The overall effect of these activities may play a key role in determining the metastatic spread of breast tumors, and therefore disease progression.

The present findings on the interplay between tumor cells, stroma/inflammatory cells and inflammatory cytokines/ chemokines probably represent only the tip of the iceberg. Additional levels of cross-talk between tumor cells and inflammatory mediators may establish a complex network of interactions between breast transformed cells and their microenvironment, eventually affecting the malignant process. Better understanding of the role played by the inflammatory components in breast cancer progression may lead the way to the design of therapeutic manipulations. Specific targeting (by antibodies, chemokine antagonists, etc.), primarily of well-identified deleterious inflammatory mediators, may block the activity of harmful cascades that are induced by these factors. In addition, inhibition of factors that are involved in site-specific metas- 


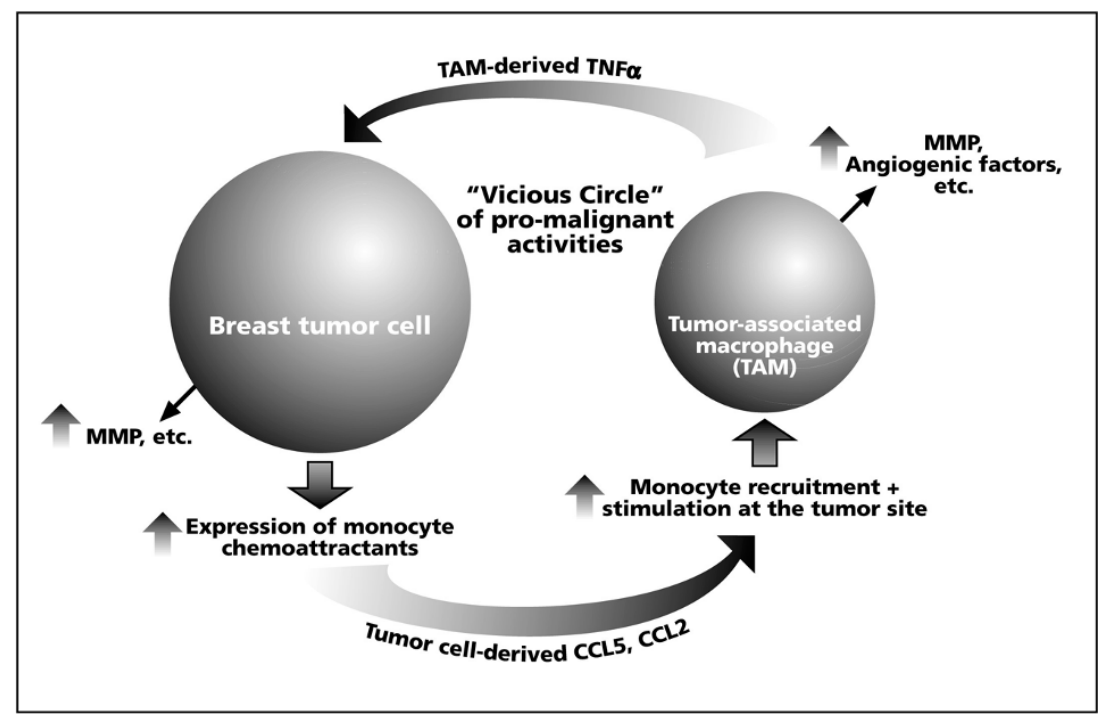

A proposed model for the potential role of the interactions between tumor cells and inflammatory elements in breast cancer progression. The expression of monocyte chemoattractants (CCL5 and CCL2) by breast tumor cells may induce monocyte infiltration to breast tumor sites. The resulting tumor-associated macrophages (TAM) may express promalignant mediators, such as tumor necrosis factor alpha (TNF- $\alpha$ ). This inflammatory cytokine may further promote the expression of tumor-supporting factors by the tumor cells, including matrix metalloproteinases (MMP) and the monocyte chemoattractants CCL5 and CCL2. The elevated expression of these chemokines by the tumor cells may result in additional monocyte recruitment, and in the stimulation of TAM at the tumor site. TAM stimulation may give rise to promoted levels of expression of promalignant factors, such as MMP, angiogenic mediators and TNF- $\alpha$. Some of these activities may be stimulated directly by the chemokines. TAM-derived TNF- $\alpha$ may in turn further increase the expression of monocyte chemoattractants (e.g. CCL5, CCL2) by the tumor cells, and so on. This process may be aided by other functions of inflammatory cells/cytokines/chemokines (vascularization, release of growth factors, etc.; see Table 1) that eventually support the growth of the primary tumor and distant metastasis formation (possibly assisted by other chemokines, such as CXCL12).

tasis formation (e.g. CXCL12) may reduce the metastatic spread of the tumor cells. These approaches could be complemented by overexpression of potential antimalignant factors (such as angiostatic, CD4+/natural killer cellattracting chemokines).

The combined effects of such manipulations may result in restriction of breast cancer development, and possibly of metastasis formation. However, one should take into account that the intact activity of immune mechanisms may be impaired by the use of such approaches. Further research should therefore be conducted in order to identify the exact role of each mediator, alone and in conjunction with others, in breast cancer progression. Better understanding of the interrelationships between tumor cells and their surrounding may lead the way for well-

This article is the first in a review series on Host microenvironment in breast cancer development, edited by Gloria Heppner.

Other articles in the series can be found at http://breast-cancer-research.com/ articles/series.asp?rqs=heppner controlled usage of specific manipulations that may eventually limit breast cancer progression.

\section{Acknowledgements}

The author would like to thank Prof. Keydar, Prof. Witz, Prof. Chaitchik, Dr Wigler, Dr Ran, Ms Azenshtein, Ms Luboshits, Mr Neumark and Ms Shina for their contribution.

\section{References}

1. Stewart THM, Heppner GH: Immunological enhancement of breast cancer. Parasitology 1997, 115:S141-S153.

2. Hadden JW: The immunology and immunotherapy of breast cancer: an update. Int J Immunopharmacol 1999, 21:79-101.

3. Mantovani A, Bottazzi B, Colotta F, Sozzani S, Ruco L: The origin and function of tumor-associated macrophages. Immunol Today 1992, 13:265-270.

4. Leek RD, Lewis CE, Whithouse R, Greenall M, Clarke J, Harris $\mathrm{AL}$ : Association of macrophage infiltration with angiogenesis and prognosis in invasive breast carcinoma. Cancer Res 1996, 56:4625-4629.

5. Crowther M, Brown NJ, Bishop ET, Lewis CE: Microenvironmental influence on macrophage regulation of angiogenesis in wounds and malignant tumors. J Leuk Biol 2001, 70:478-490.

6. van Netten JP, Ashmead BJ, Cavers D, Fletcher C, Thornton IG, Antonsen BL, Coy P, Brigden ML: 'Macrophages' and their putative significance in human breast cancer. $\mathrm{Br} J$ Cancer 1992, 66:220-221.

7. O'Sullivan C, Lewis CE: Tumour-associated leucocytes: friends or foes in breast carcinoma. J Pathol 1994, 172:229-235.

8. Balkwill F, Mantovani A: Inflammation and cancer: back to Virchow? Lancet 2001, 357:539-545. 
9. Balkwill F: Tumor necrosis factor or tumor promoting factor? Cytokine Growth Factor Rev 2002, 13:135-141.

10. Leek RD, Landers R, Fox SB, Ng F, Harris AL, Lewis CE: Association of tumour necrosis factor alpha and its receptors with thymidine phosphorylase expression in invasive breast carcinoma. Br J Cancer 1998, 77:2246-2251.

11. Miles DW, Happerfield LC, Naylor MS, Bobrow LG, Rubens RD, Balkwill FR: Expression of tumour necrosis factor (TNF- $\alpha$ ) and its receptors in benign and malignant breast tissue. Int $J$ Cancer 1994, 56:777-782.

12. Sheen-Chen S-M, Chen W-J, Eng H-L, Chou F-F: Serum concentration of tumor necrosis factor in patients with breast cancer. Breast Cancer Res Treat 1997, 43:211-215.

13. Karczewska A, Nawrocki S, Breborowicz D, Filas V, Mackiewicz A: Expression of interleukin-6, interleukin-6 receptor, and glycoprotein 130 correlates with good prognoses for patients with breast carcinoma. Cancer 2000, 88:2061-2071.

14. Kurebayashi J: Regulation of interleukin-6 secretion from breast cancer cells and its clinical implications. Breast Cancer 2000, 7:124-129.

15. Jin L, Yuan RQ, Fuchs A, Yao Y, Joseph A, Schwall R, Schnitt SJ, Guida A, Hastings HM, Andres J, Turkel, G, Polverini PJ, Goldberg ID, Rosen EM: Expression of interleukin-1 $\beta$ in human breast carcinoma. Cancer 1997, 80:421-434.

16. Sallusto $F$, Mackay $C R$, Lanzavecchia A: The role of chemokine receptors in primary, effector, and memory immune responses. Annu Rev Immunol 2000, 18:593-620.

17. Muller A, Homey B, Soto H, Ge N, Catron D, Buchanan ME, McClanahan T, Murphy E, Yuan W, Wagner SN, Barrera JL, Mohar $A$, Verástegul E, Zlotnik A: Involvement of chemokine receptors in breast cancer metastasis. Nature 2001, 410:50-56.

18. Saji H, Koike M, Yamori T, Saji S, Seiki M, Matsushima K, Toi M: Significant correlation of monocyte chemoattractant protein-1 expression with neovascularization and progression of breast carcinoma. Cancer 2001, 92:1085-1091.

19. Ueno $T$, Toi $M$, Saji $H$, Muta $M$, Bando $H$, Kuroi $K$, Koike $M$, Inadera $\mathrm{H}$, Matsushima $\mathrm{K}$ : Significance of macrophage chemoattractant protein-1 in macrophage recruitment, angiogenesis, and survival in human breast cancer. Clin Cancer Res 2000. 6:3282-3289.

20. Goede V, Brogelli L, Ziche M, Augustin HG: Induction of inflammatory angiogenesis by monocyte chemoattractant protein-1. Int J Cancer 1999, 82:765-770.

21. Salcedo R, Ponce ML, Young HA, Wasserman K, Ward JM, Kleiman HK, Oppenheim JJ, Murphy WJ: Human endothelial cells express CCR2 and respond to MCP-1: direct role of MCP-1 in angiogenesis and tumor progression. Blood 2000, 96:34-40.

22. Luboshits G, Shina S, Kaplan O, Engelberg S, Nass D, LifshitzMercer B, Chaitchik S, Keydar I, Ben-Baruch A: Elevated expression of the CC chemokine regulated on activation, normal $\mathrm{T}$ cell expressed and secreted (RANTES) in advanced breast carcinoma. Cancer Res 1999, 59:4681-4687.

23. Niwa $Y$, Akamatsu H, Niwa $H$, Sumi H, Ozaki Y, Abe A: Correlation of tissue and plasma RANTES levels with disease course in patients with breast or cervical cancer. Clin Cancer Res 2001, 7:285-289.

24. Mira E, Lacalle RA, González MA, Gomez-Mouton C, Abad JL, Bernad A, Martínez-A C, Manes S: A role for chemokine receptor transactivation in growth factor signaling. EMBO Reports 2001, 2:151-156.

25. Azenshtein E, Luboshits G, Shina S, Neumark E, Shahbazian D, Weil M, Wigler N, Keydar I, Ben-Baruch A: The CC chemokine RANTES in breast carcinoma progression: regulation of expression and potential mechanisms of promalignant activity. Cancer Res 2002, 62:1093-1102.

26. Klier CM, Nelson EL, Cohen CD, Horuk R, Schlondorff D, Nelson PJ: Chemokine-induced secretion of gelatinase $B$ in primary human monocytes. Biol Chem 2001, 382:1405-1410.

27. Ali S, Kaur J, Patel KD: Intercellular cell adhesion molecule-1, vascular cell adhesion molecule-1, and regulated on activation normal $T$ cell expressed and secreted are expressed by human breast carcinoma cells and support eosinophil adhesion and activation. Am J Pathol 2000, 157:313-321.

28. Inadera H, Sekiya T, Yoshimura T, Matsushima K: Molecular analysis of the inhibition of monocyte chemoattractant protein-1 gene expression by estrogens and xenoestrogens in MCF-7 cells. Endocrinology 2000, 141:50-59.
29. Youngs SJ, Ali SA, Taub DD, Rees RC: Chemokines induce migrational responses in human breast carcinoma cell lines. Int J Cancer 1997, 71:257-266.

30. Dorsey R, Kundu N, Yang Q, Tannenbaum CS, Sun H, Hamilton TA, Fulton AM: Immunotherapy with interleukin-10 depends on the CXC chemokines inducible protein-10 and monokine induced by IFN- $\gamma$. Cancer Res 2002, 62:2606-2610.

31. Braun SE, Chen, K, Foster RG, Kim CH, Hromas R, Kaplan MH, Broxmeyer HE, Cornetta K: The CC chemokine CK $\beta-11 /$ MIP$3 \beta / E L C / E x o d u s ~ 3$ mediates tumor rejection of murine breast cancer cells through NK cells. J Immunol 2000, 164:40254031.

32. Belperio JA, Keane MP, Arenberg DA, Addison CL, Ehlert JE, Burdick MD, Strieter RM: CXC chemokines in angiogenesis. $J$ Leukoc Biol 2000, 68:1-8.

33. De Larco JE, Wuertz BRK, Rosner KA, Erickson SA, Gamache DE, Carlos Manivel C, Furcht LT: A potential role for interleukin8 in the metastatic phenotype of breast carcinoma cells. $\mathrm{Am} \mathrm{J}$ Pathol 2001, 158:639-646.

34. Miller LJ, Kurtzman SH, Wang Y, Anderson $\mathrm{KH}$, Lindquist RR, Kreutzer DL: Expression of interleukin-8 receptors on tumor cells and vascular endothelial cells in human breast cancer tissue. Anticancer Res 1998, 18:77-82.

35. Green AR, Green VL, White MC, Speirs V: Expression of cytokine messenger RNA in normal and neoplastic human breast tissue: identification of interleukin-8 as a potential regulatory factor in breast tumours. Int $J$ Cancer 1997, 72:937941.

\section{Correspondence}

A Ben-Baruch, Department of Cell Research and Immunology, George S Wise Faculty of Life Sciences, Tel-Aviv University, Tel-Aviv 69978, Israel. Tel: +972 3640 7933; fax: +972 3642 2046; e-mail: aabb@post.tau.ac.il 